\title{
Prognosis of early stage pulmonary mucinous adenocarcinoma with different treatments
}

\author{
Jinyuan Liu, Shijiang Zhang, Jinhua Luo \\ Department of Thoracic Surgery, Jiangsu Province Hospital, the First Affiliated Hospital of Nanjing Medical University, Nanjing, China \\ Contributions: (I) Conception and design: S Zhang, J Luo; (II) Administrative support: J Luo; (III) Provision of study materials or patients: J Liu; (IV) \\ Collection and assembly of data: J Liu, S Zhang; (V) Data analysis and interpretation: J Liu, S Zhang; (VI) Manuscript writing: All authors; (VII) \\ Final approval of manuscript: All authors. \\ Correspondence to: Shijiang Zhang, MD; Jinhua Luo, MD. Department of Cardiothoracic Surgery, Jiangsu Province Hospital, the First Affiliated Hospital \\ of Nanjing Medical University, No. 300 Guangzhou Road, Nanjing 210029, China. Email: shijiangzhang@hotmail.com; 38281125@qq.com.
}

Background: The effects of surgical approach and adjuvant chemotherapy (AC) of early stage pulmonary mucinous adenocarcinoma (MAD) have not been thoroughly studied yet. This study intends to clarify whether AC provides clinical benefit to the early stage MAD patients and the survival difference between surgical approaches.

Methods: All cases of stage I MAD were identified from the SEER database during the period of 20092014. The primary cohort was divided into AC and surgery (S) groups. Meanwhile, the patients with tumor $\leq 1 \mathrm{~cm}$ were divided into lobectomy and sublobar resection group. Clinical characteristics, treatments and survival data including overall survival (OS) and cancer-specific survival (CSS) were analyzed.

Results: A total of 1,816 patients were included in the final cohort. Referring to surgical procedure, 140 patients received lobectomy and 75 patients received sublobar resection. AC showed worse survival outcomes than surgery alone (OS: 71.2 vs. 93.4 months; CSS: 74.9 vs. 101.1 months). No significant difference was observed between lobectomy group and sublobar resection group (OS: 97.3 vs. 93.1 months; CSS: 103.7 vs. 101.3 months). Consistent results were also shown after the propensity score matching analysis (PSM) was applied.

Conclusions: Early stage of MAD has an ideal prognosis. AC may bring adverse effects which would lower OS and CSS of stage I MAD patients. No significant difference is observed in the comparison of prognosis between lobectomy and sublobar resection in tumor size $\leq 1 \mathrm{~cm}$ MAD patients.

Keywords: Mucinous adenocarcinoma (MAD); lung cancer; SEER; prognosis; treatment

Submitted Jan 04, 2020. Accepted for publication Jul 08, 2020.

doi: $10.21037 /$ tcr-20-194

View this article at: http://dx.doi.org/10.21037/tcr-20-194

\section{Introduction}

Lung cancer is still the leading cause of cancer-related death in the world. Besides, the prevalence of lung cancer is increasingly high. It can be classified as non-small cell lung cancer (NSCLC) and small cell lung cancer (SCLC), which accounts for $80-85 \%$ and $20 \%$ (1). Among NSCLC, adenocarcinoma (AD) is the majority type. Mucinous adenocarcinoma (MAD) is one of the rare subtypes in $\mathrm{AD}$ which accounts for only $5-6 \%$ (2). MAD is diagnosed according to the pathological and morphological features with abundant intracytoplasmic mucin and columnar or goblet cancer cells (3). Travis et al. had also reported that MAD tended to develop as multicentric or multilobar pathologically (4). Because of its rare prevalence, the clinical and biological features have not been fully studied $(2,5)$. It had been reported that the MAD was associated with low incidence of EGFR mutation and high KRAS mutation rate, which would be primarily resistant to EGFR tyrosine kinase inhibitors (EGFR-Tkis) (6-8). Furthermore, its prognosis 
had not been fully studied. Some previous studies showed that MAD had an adverse prognosis (9-12). Nevertheless, other studies reported an ideal clinical outcome of MAD comparing to non-MAD patients (13).

A new classification system of lung $\mathrm{AD}$ has been published in 2011 by the International Association for the Study of Lung Cancer (IASLC), American Thoracic Society (ATS) and European Respiratory Society (ERS). Besides $M A D$, the non-MADs were categorized to five different subtypes according to their pathological characteristics including acinar, lepidic, papillary, micro-papillary and solid predominant types (2). Since the classification system was proposed, a large amount of studies had been showed the relation between pathological subtypes and their prognosis. Patients with lepidic predominant had the best prognosis, while acinar and papillary had worse prognosis than lepidic. Patients with micropapillary and solid predominant had the worst prognosis for a relatively high recurrent rate. Besides, numerous studies of treatment strategies in each subtype had also been performed and reported. Surgery was the first choice in the early stage of each predominant pattern patients, while adjuvant chemotherapy (AC) was highly recommended in micropapillary and solid predominant $(14,15)$. Compared to non-MAD, few studies had been reported the prognosis and treatment strategy of MAD especially the early stage of MAD. The necessity of AC had not been verified on the early stage MAD patients. Additionally, there is still no consensus on the standard surgical approach for the patients with less than $1 \mathrm{~cm}$ tumors.

As a result, we intend to demonstrate the clinical characteristics of MAD and identify different prognostic factors based on Surveillance, Epidemiology, and End Result (SEER) database. The effect of AC and different surgical approaches on the early stage MAD patients would be analyzed. We present the following article in accordance with the STROBE reporting checklist (available at http:// dx.doi.org/10.21037/tcr-20-194).

\section{Methods}

\section{Data source}

The patients diagnosed as stage $\mathrm{I} A \mathrm{D}$ in the primary site of main bronchus and lung (SEER primary site code $=$ C340 - C349) were identified. The clinical characteristics and outcomes were extracted and analyzed. Cases with missing data, unknown stages or no surgery were excluded after discussion. A total of 4,789 patients were pathologically diagnosed as MAD including signet-ring cell carcinoma (SRCC), solid AD with mucin production (SA), primary mucinous bronchioloalveolar carcinoma (MBAC) and mucinous colloid adenocarcinoma (MCA). The study is consulted by the ethics board of Jiangsu Province Hospital. Considering the study would be conducted using public electronic database. The consent of the patients is not required. The request of accessing SEER database is also approved by the SEER program in National Cancer Institute. The selection process of primary cohort was demonstrated in Figure 1.

\section{Statistical analysis}

The primary outcome was overall survival (OS). The Kaplan-Meier method and log-rank test were used to demonstrate survival status of the cohort and assess the prognostic differences between various treatments. Univariate and multivariate analysis was performed incorporating gender, age, surgery, stage, AC and histological subtypes.

The Kaplan-Meier, Cox regression and propensity score matching analysis (PSM) were performed using the $\mathrm{R}$ (version 3.5.0). Hazard ratio (HR) and the $95 \%$ confident interval (95\% CI) were reported. The statistical difference was considered as significant when $\mathrm{P}<0.05$. All tests were two-sided.

\section{Result}

\section{Clinical features of primary cohort}

A total of 4789 MAD patients were identified from the database. Among them, 2,094 patients were stage I MAD according to the $6^{\text {th }}$ edition AJCC TNM stage. The final cohort contained 1,816 cases despite the those without data of surgery. In the final cohort, 1,695 patients were classified in surgery alone group (S) and 121 patients were classified in adjuvant chemotherapy group (AC). The mean age of final cohort was 66-year-old while it was 64.2 in AC group and 66.1 in $\mathrm{S}$ group. Female patient and white patients accounted for a larger proportion of all patients, which were $59.6 \%$ and $83.6 \%$ respectively.

A total of 1,368 patients (75.3\%) was stage IA and 448 patients was IB. In the subgroups, IB patients were 56 (46.3\%) in AC group and $392(23.1 \%)$ in S group. The mean tumor size was $21.8 \mathrm{~mm}$ in all patients. Patients in AC group had larger tumor size than $\mathrm{S}$ group (26.3 vs. $21.5 \mathrm{~mm}, \mathrm{P}<0.001)$. In terms of pathological subtypes, 


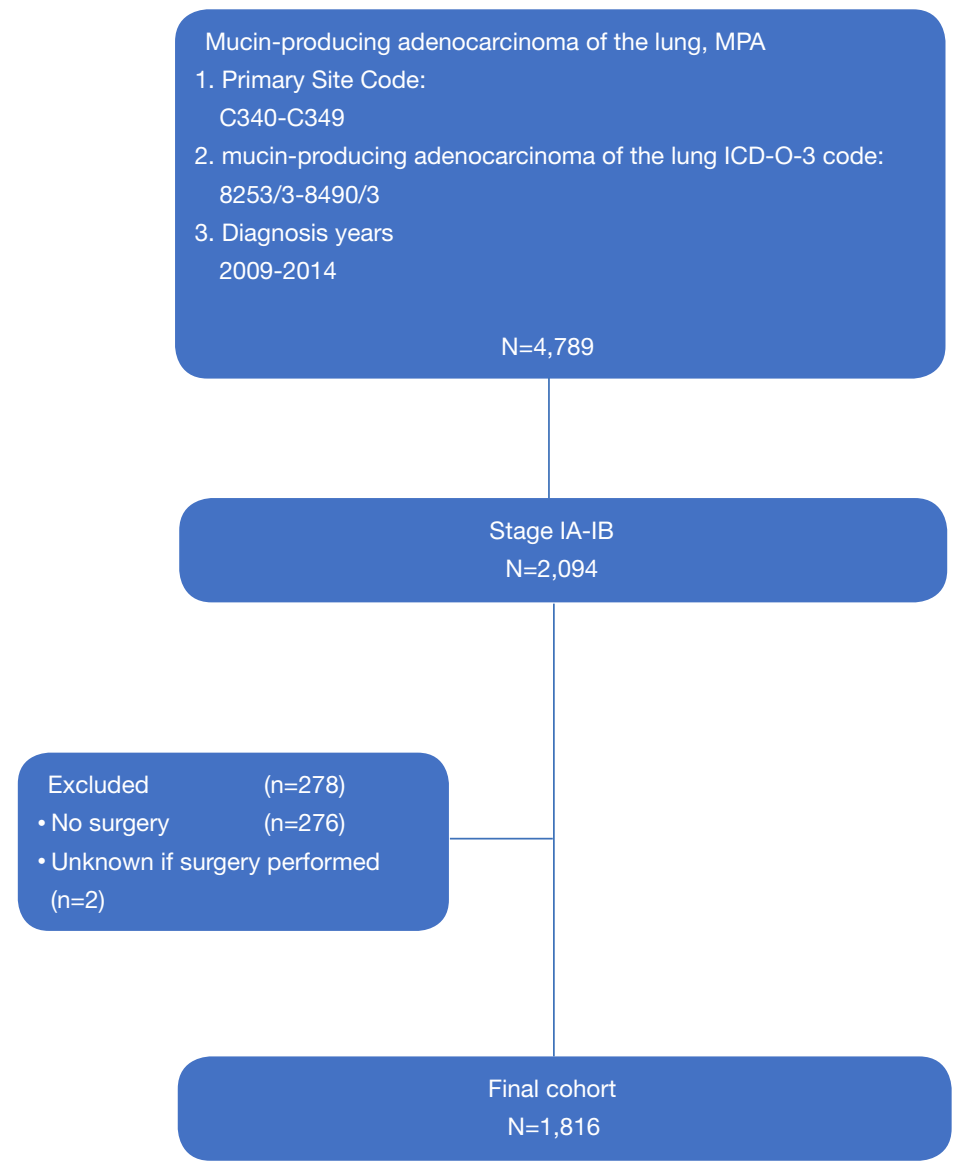

Figure 1 The selection process of primary cohort.

MAD and mucin-producing $\mathrm{AD}$ were the predominant in both groups. All patients received radical surgical treatments while 78 patients in AC groups also received postoperative radio-therapy. The clinical characteristics and oncological features were demonstrated in Table 1 .

\section{Clinical features of patients with $\leq 1 \mathrm{~cm}$ tumor}

Among the stage I MAD patients, 179 were identified as tumor size $\leq 1 \mathrm{~cm}$ whose the average tumor size was $8.2 \mathrm{~mm}$. The lobectomy group had 104 patients while the sub-lobectomy group had 75 patients. The mean ages of two groups were 64.7 and 67.4 years which was not significantly different $(\mathrm{P}=0.118)$. The mean tumor size of lobectomy group was larger than the sublobar resection group (8.5 vs. $7.7 \mathrm{~mm}, \mathrm{P}=0.025)$. Female patients accounted for a larger proportion in both groups ( $73 \%$ and $44 \%)$. In accordance with the primary cohort, white patients and MAD were the predominant race and pathological subtype. A number of
2 patients received chemotherapy in sub-lobectomy group while 4 patients in lobectomy group received chemotherapy (Table 2).

\section{Survival analysis of subgroups}

The OS and CSS of $S$ group were both superior to AC group (OS: 93.4 vs. 71.2 months, $\mathrm{P}<0.001$; CSS: 101.1 vs. 74.9 months, $\mathrm{P}<0.001$ ) (Figure $2 A, B)$. In terms of the patients with tumor size $<1 \mathrm{~cm}$, the sublobar resection which included wedge resection and segmentectomy had similar OS and CSS with the lobectomy group (OS: 93.1 vs. 97.3 months, $\mathrm{P}=0.65$; CSS: 101.3 vs. 103.7 months, $\mathrm{P}=0.809$ ) (Figure $2 C, D$ ). In order to minimize the influence to the prognostic prediction from the difference of clinical characteristics, the PSM analysis with 3:1 matching was therefore performed. The characteristics of patients in postmatched groups and results were demonstrated in Table S1,S2 and Figures S1,S2. The result was concordant to the result 
Table 1 Demographic and clinicopathologic variables in the patients with stage I mucinous adenocarcinoma

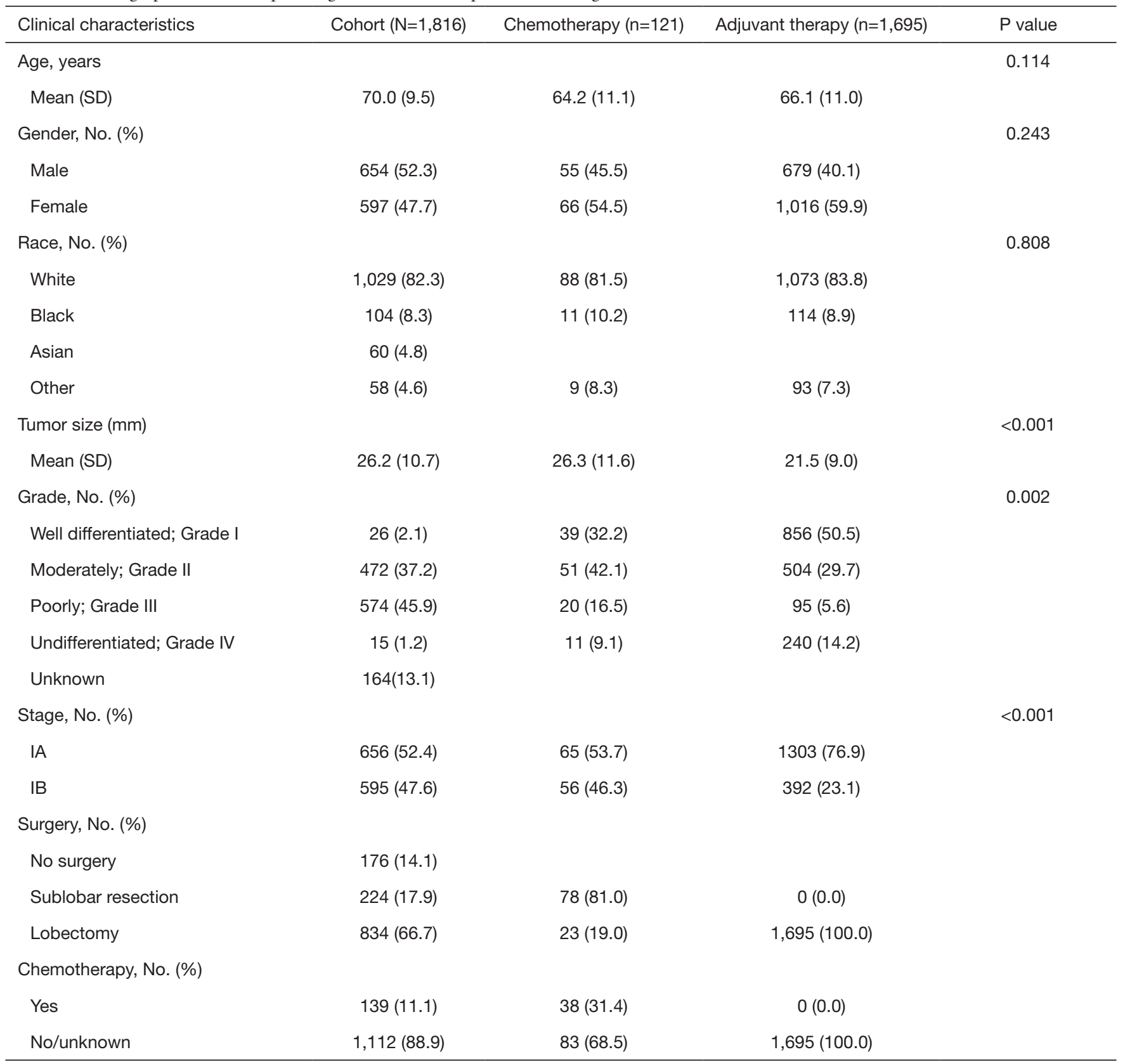

of primary cohort indicating that patients in adjuvant chemotherapy group had worse outcomes (OS: 116 vs. 69 months, $\mathrm{P}=0.0022$ ) (Figure S3, Table S3).

\section{Discussion}

Pulmonary MAD is a special pathological subtype of AD. Previous reports showed a relatively malignant feature and unideal prognosis of MAD comparing to other types of $\operatorname{AD}(2,9,10,11)$. Few studies reported the treatments and prognosis of early stage of MAD. To fill the gap of knowledge in treatment for early stage of MAD, we therefore conducted this study. Regarding to its low incidence, we chose patients from SEER database which was sponsored by National Cancer Institute and covered approximately $28 \%$ of US population. In our study, we 
Table 2 Demographic and clinic pathologic variables in the mucinous adenocarcinoma patients with tumor size $\leq 1 \mathrm{~cm}$

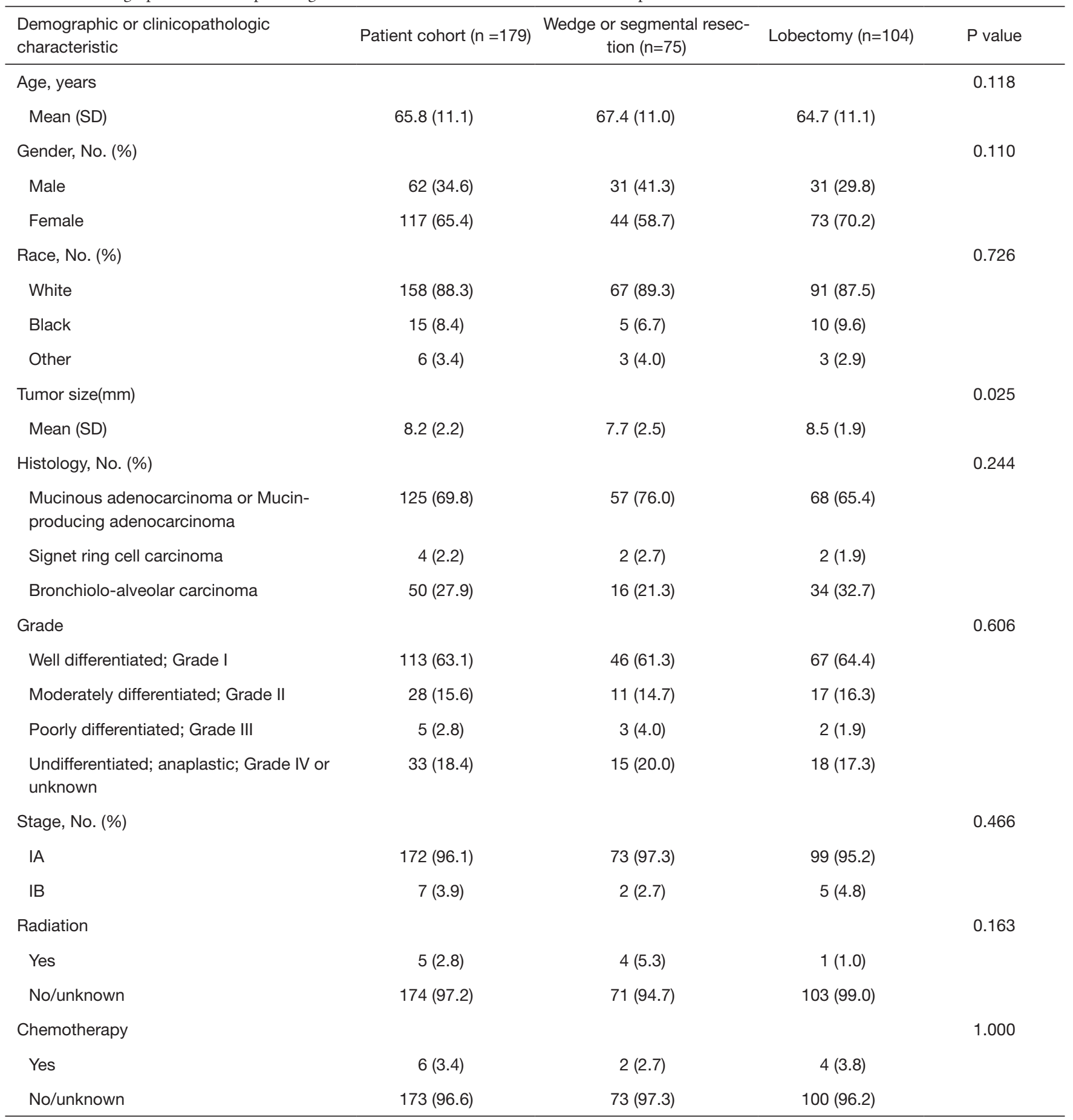

analyzed the effect of adjuvant chemotherapy in stage I MAD patients and survival outcomes of different surgical approaches.

Previous studies showed adjuvant chemotherapy provided little benefits to early stage of NSCLC (13-16). Principally, patients in stage IA were not recommended to receive adjuvant chemotherapy, while its effect and necessity in stage IB was unclear (16). Based on the 
A

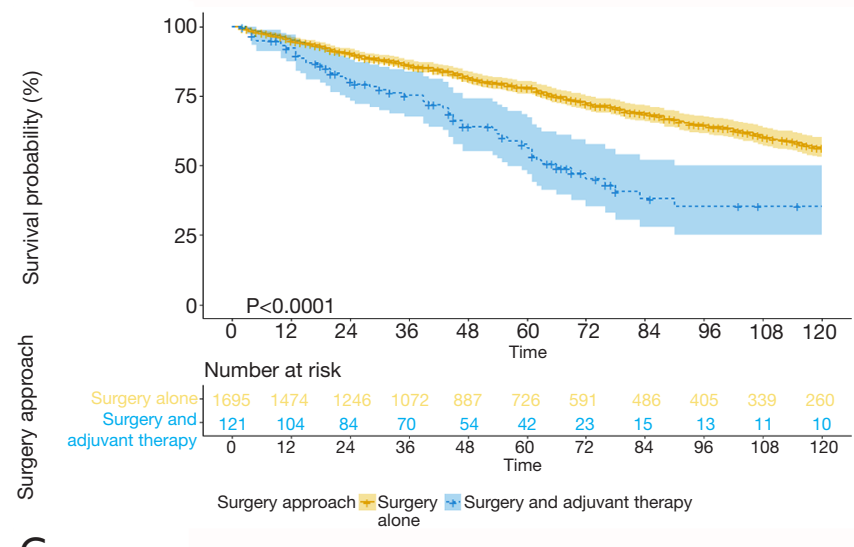

C

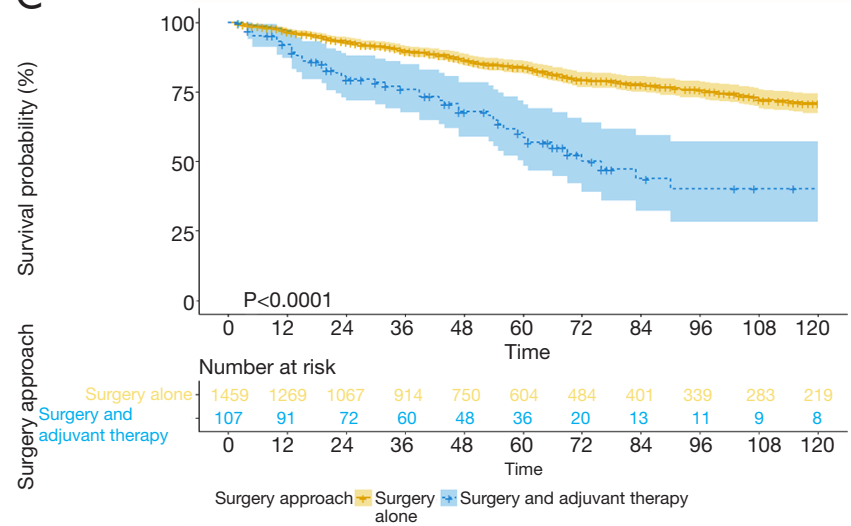

B

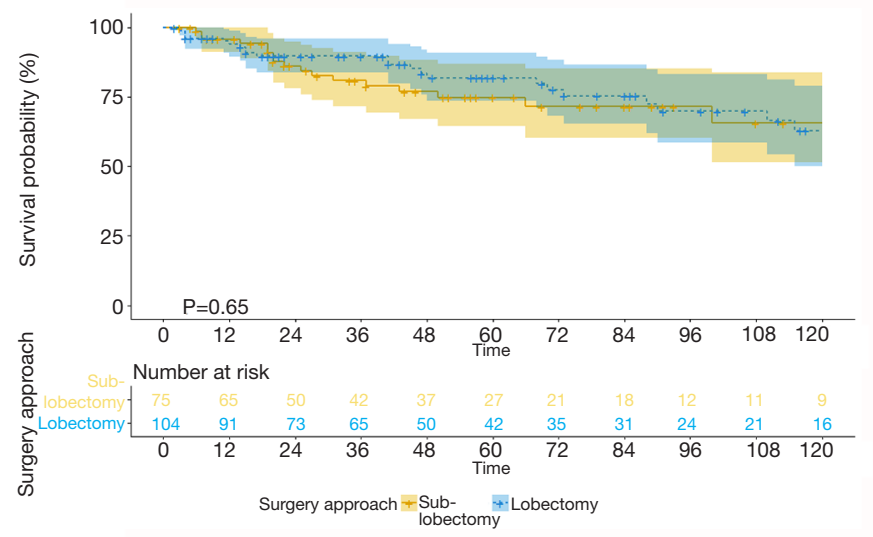

$\mathrm{D}$

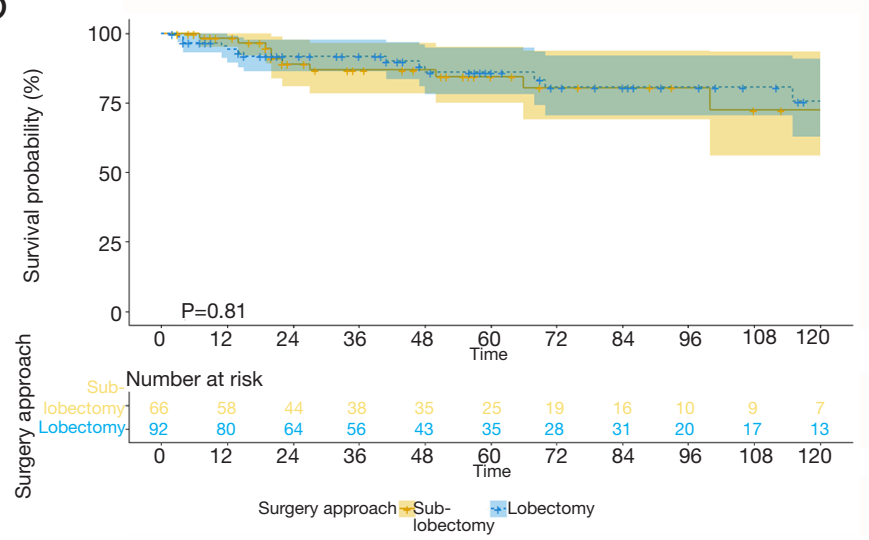

Figure 2 The overall survival and cancer-specific survival of primary cohort and the patients with tumor size $\leq 1 \mathrm{~cm}$; (A) The overall survival of primary cohort; (C) the overall survival of patients with tumor size $\leq 1 \mathrm{~cm}$; (B) the cancer-specific survival of primary cohort; (D) the cancer-specific survival of patients with tumor size $\leq 1 \mathrm{~cm}$.

molecular features of high KRAS mutation rate, MAD was considered as one of the malignancies of poor prognosis with lower OS and higher rate of tumor recurrence $(17,18)$. In this study, it showed that adjuvant chemotherapy even provided worse prognostic outcomes. The heterogeneity of clinical features in two groups may account for the results. The mean tumor sizes in AC group was significantly larger than $\mathrm{S}$ group, which had been proved to be a negative prognostic factor. Besides, AC group included more patients in worse differentiated grades and IB stage. These characteristic differences mostly accounted for the worse prognosis. It indicated that most of the clinical doctors would not prescribe adjuvant chemotherapy for the stage I MAD patients. Nevertheless, they might tend to administer adjuvant chemotherapies to these patients with potentially worse outcomes. In order to exclude the effects from the characteristics, the PSM analysis was also performed. It also showed similar results as the primary cohort.
In terms of MAD patients with tumor size $\leq 1 \mathrm{~cm}$, the result indicated that lobectomy group had similar survival outcomes to sublobar resection group. Although Dai et al. had reported lobectomy would have ideal prognosis than sublobar resection in a large scale of early stage of NSCLC (19), MAD patients were the exceptions. On the other hand, some patients with poor cardiopulmonary condition or other comorbidities were tend to receive compromised sublobar resection, which could spare as much lung tissue as possible (20-23). Therefore, no significant difference would be observed in OS. In order to investigate the prognosis associated with cancer despite other conditions, we therefore studied the cancer-specific survival. It showed no difference between two surgical methods. It might attribute to the ideal prognosis of early stage MAD. The median survival of both subgroups exceeded 120 months, which were consistent with the previous studies.

Limitations have to be admitted in this study. Firstly, 
MAD is a rare pathological subtype of NSCLC. The final cohort contains 1,816 MAD patients which is a relatively small sample size. Among them, only 121 patients are included in AC group. Given the fact that it is a retrospective study, clinical characteristics of each groups are difficult to unify. It might bring bias to the final results. Secondly, PSM was used in the study to minimize the different characteristics. However, some cases would be deleted in the process and make the final result over-optimized. Last, although SEER database contains tremendous amount of data, the integrity could be improved. Some data of surgical procedure and tumor features is not available in cases during the selection process, which we therefore have to discard. Moreover, neither comorbidity data nor tumor recurrence was recorded in the database. As a result, progression-free survival could not be calculated in this study.

In summary, adjuvant chemotherapy is not helpful in the stage I MAD patients in our study. No significant difference is observed in the comparison of prognosis between lobectomy and sublobar resection in tumor size $\leq 1 \mathrm{~cm}$ MAD patients. A larger cohort with more complete data is warranted to further investigate the necessity of adjuvant chemotherapy in early stage MAD and the preferable surgical approach.

\section{Acknowledgments}

Funding: None.

\section{Footnote}

Reporting Checklist: The authors have completed the STROBE reporting checklist. Available at http://dx.doi. org/10.21037/tcr-20-194

Conflicts of Interest: All authors have completed the ICMJE uniform disclosure form (available at: http://dx.doi. org/10.21037/tcr-20-194). The authors have no conflicts of interest to declare.

Ethical Statement: The authors are accountable for all aspects of the work in ensuring that questions related to the accuracy or integrity of any part of the work are appropriately investigated and resolved. The study is consulted by the ethics board of Jiangsu Province Hospital. The consent of the patients is not required.
Open Access Statement: This is an Open Access article distributed in accordance with the Creative Commons Attribution-NonCommercial-NoDerivs 4.0 International License (CC BY-NC-ND 4.0), which permits the noncommercial replication and distribution of the article with the strict proviso that no changes or edits are made and the original work is properly cited (including links to both the formal publication through the relevant DOI and the license). See: https://creativecommons.org/licenses/by-nc-nd/4.0/.

\section{References}

1. Bueno R, Hughes E, Wagner S, et al. Validation of a molecular and pathological model for five-year mortality risk in patients with early stage lung adenocarcinoma. J Thorac Oncol 2015;10:67-73.

2. Travis WD, Brambilla E, Noguchi M, et al. International association for the study of lung cancer/american thoracic society/european respiratory society international multidisciplinary classification of lung adenocarcinoma. J Thorac Oncol 2011;6:244-85.

3. Truini A, Santos Pereira P, Cavazza A, et al. Classification of different patterns of pulmonary adenocarcinomas. Expert Rev Respir Med 2015;9:571-86.

4. Travis WD, Costabel U, Hansell DM, et al. An official American Thoracic Society/European Respiratory Society statement: Update of the international multidisciplinary classification of the idiopathic interstitial pneumonias. Am J Respir Crit Care Med 2013;188:733-48.

5. Shim HS, Kenudson M, Zheng Z, et al. Unique Genetic and Survival Characteristics of Invasive Mucinous Adenocarcinoma of the Lung. J Thorac Oncol 2015;10:1156-62.

6. Hata A, Katakami N, Fujita S, et al. Frequency of EGFR and KRAS Mutations in Japanese Patients with Lung Adenocarcinoma with Features of the Mucinous Subtype of Bronchioloalveolar Carcinoma. J Thorac Oncol 2010;5:1197-200.

7. Ichinokawa H, Ishii G, Nagai K, et al. Distinct clinicopathologic characteristics of lung mucinous adenocarcinoma with KRAS mutation. Hum Pathol 2013;44:2636-42.

8. Zheng D, Wang R, Zhang Y, et al. The prevalence and prognostic significance of KRAS mutation subtypes in lung adenocarcinomas from Chinese populations. Onco Targets Ther 2016;9:833-43.

9. Cai D, Li H, Wang R, et al. Comparison of clinical 
features, molecular alterations, and prognosis in morphological subgroups of lung invasive mucinous adenocarcinoma. Onco Targets Ther 2014;7:2127-32.

10. Lee HY, Cha MJ, Lee KS, et al. Prognosis in Resected Invasive Mucinous Adenocarcinomas of the Lung: Related Factors and Comparison with Resected Nonmucinous Adenocarcinomas. J Thorac Oncol 2016;11:1064-73.

11. Yanagawa N, Shiono S, Abiko M, et al. New IASLC/ATS/ ERS classification and invasive tumor size are predictive of disease recurrence in stage I lung adenocarcinoma. J Thorac Oncol 2013;8:612-8.

12. Warth A, Muley T, Meister M, et al. The Novel Histologic International Association for the Study of Lung Cancer/ American Thoracic Society/European Respiratory Society Classification System of Lung Adenocarcinoma Is a Stage-Independent Predictor of Survival. J Clin Oncol 2012;30:1438-46.

13. Tsao MS, Marguet S, Le Teuff G, et al. Subtype Classification of Lung Adenocarcinoma Predicts Benefit From Adjuvant Chemotherapy in Patients Undergoing Complete Resection. J Clin Oncol 2015;33:3439-46.

14. Russell PA, Wainer Z, Wright GM, et al. Does Lung Adenocarcinoma Subtype Predict Patient Survival? A Clinicopathologic Study Based on the New International Association for the Study of Lung Cancer/American Thoracic Society/European Respiratory Society International Multidisciplinary Lung Adenocarcinoma Classification. J Thorac Oncol 2011;6:1496-504.

15. Coutinho D, Goncalves A, Antunes A, et al. Adjuvant chemotherapy in stage IB non-small cell lung carcinoma: A survival analysis. Rev Port Pneumol 2016;22:123-5.

16. He J, Shen J, Yang C, et al. Adjuvant Chemotherapy for the Completely Resected Stage IB Nonsmall Cell Lung

Cite this article as: Liu J, Zhang S, Luo J. Prognosis of early stage pulmonary mucinous adenocarcinoma with different treatments. Transl Cancer Res 2020;9(9):5182-5189. doi: 10.21037/ tcr-20-194
Cancer. Medicine (Baltimore) 2015;94:e903.

17. Massarelli E, Varella-Garcia M, Tang XM, et al. KRAS mutation is an important predictor of resistance to therapy with epidermal growth factor receptor tyrosine kinase inhibitors in non-small cell lung cancer. Clin Cancer Res 2007;13:2890-6.

18. Marabese M, Ganzinelli M, Garassino MC, et al. KRAS mutations affect prognosis of non-small-cell lung cancer patients treated with first-line platinum containing chemotherapy. Oncotarget 2015;6:34014-22.

19. Dai C, Shen J, Ren Y, et al. Choice of Surgical Procedure for Patients With Non-Small-Cell Lung Cancer $<=1 \mathrm{~cm}$ or $>1$ to $2 \mathrm{~cm}$ Among Lobectomy, Segmentectomy, and Wedge Resection: A Population-Based Study. J Clin Oncol 2016;34:3175-82.

20. Xue W, Duan G, Zhang X, et al. Meta-analysis of segmentectomy versus wedge resection in stage IA non-small-cell lung cancer. Onco Targets Ther 2018;11:3369-75.

21. Yin C, Zhao M, Roy S, et al. Comparison of VideoAssisted Thoracic Surgery Segmentectomy (Vats-S) Versus Video-Assisted Thoracic Surgery Lobectomy (Vats-L) for Stage I Non-Small Cell Lung Cancer (Nsclc-I) in Chinese Patients: A Systemic Review and Meta-Analysis. Value Health 2018;21:S18.

22. Zhang L, Ma W, Li Y, et al. Comparative study of the anatomic segmentectomy versus lobectomy for clinical stage I-A peripheral lung cancer by video assistant thoracoscopic surgery. J Cancer Res Ther 2013;9:S106-9.

23. Whitson BA, Groth SS, Andrade RS, et al. Survival After Lobectomy Versus Segmentectomy for Stage I Non-Small Cell Lung Cancer: A Population-Based Analysis. Ann Thorac Surg 2011;92:1943-50. 


\section{Supplementary}

Table S1 Mucinous adenocarcinoma of the lung patients survival analysis

\begin{tabular}{|c|c|c|c|c|c|c|}
\hline & \multicolumn{3}{|c|}{ Overall survival } & \multicolumn{3}{|c|}{ Cancer cause survival } \\
\hline & Mean (months) & $95 \% \mathrm{Cl}$ & $P$ value & Mean (months) & $95 \% \mathrm{Cl}$ & $P$ value \\
\hline $\begin{array}{l}\text { Surgery and adjuvant } \\
\text { therapy }(n=121)\end{array}$ & 71.2 & $62.4-79.9$ & $<0.001$ & 74.9 & $65.1-84.6$ & $<0.001$ \\
\hline $\begin{array}{l}\text { Surgery alone } \\
(n=1,695)\end{array}$ & 93.4 & $91.2-95.5$ & & 101.1 & $99.0-103.3$ & \\
\hline $\begin{array}{l}\text { Resection less than one } \\
\text { lobe }(n=75)\end{array}$ & 93.1 & $82.2-104.0$ & & 101.3 & $90.9-111.7$ & \\
\hline
\end{tabular}

Table S2 Demographic and clinic pathologic variables in the mucinous adenocarcinoma patients after propensity score matching

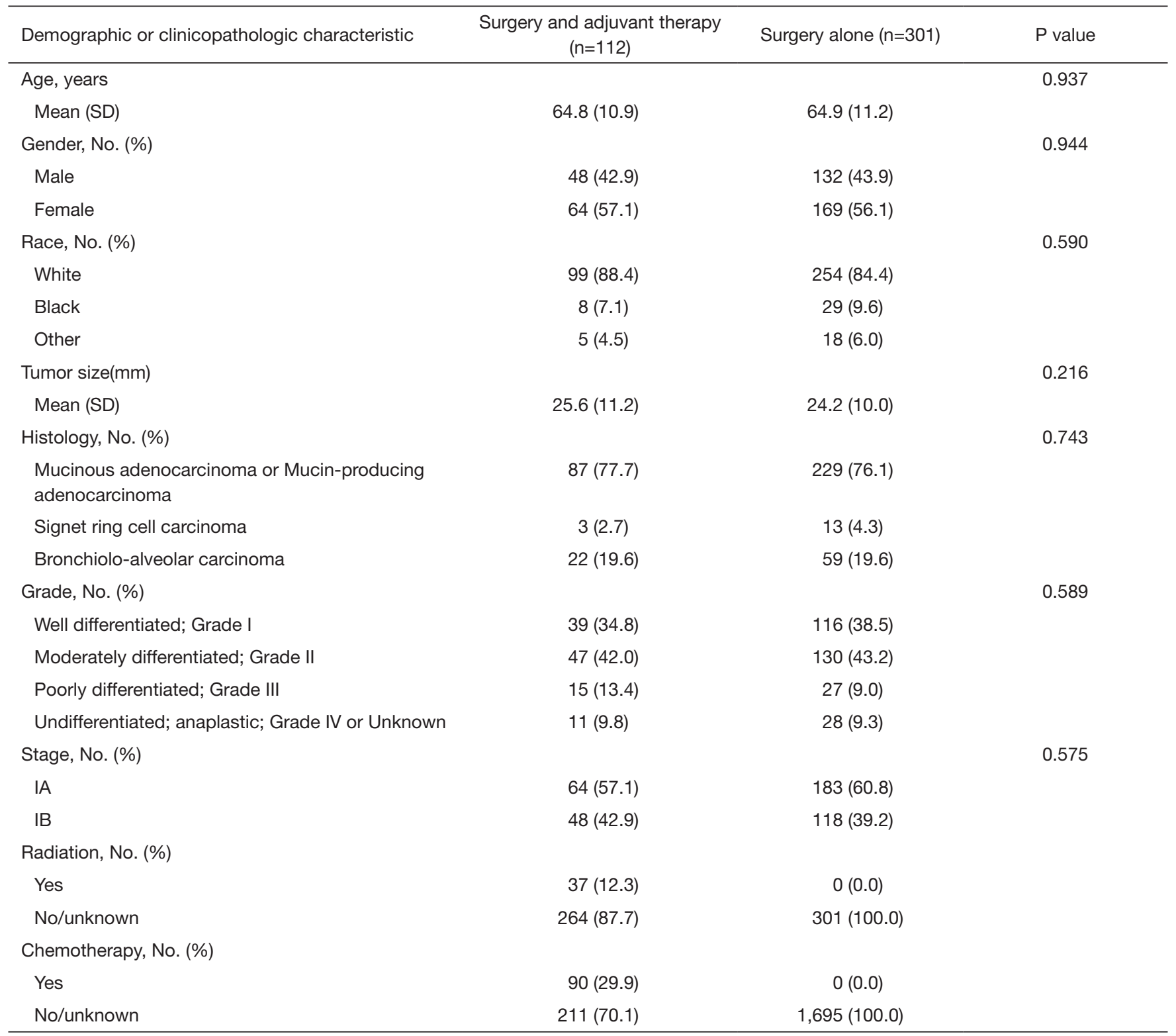


Distribution of propensity scores

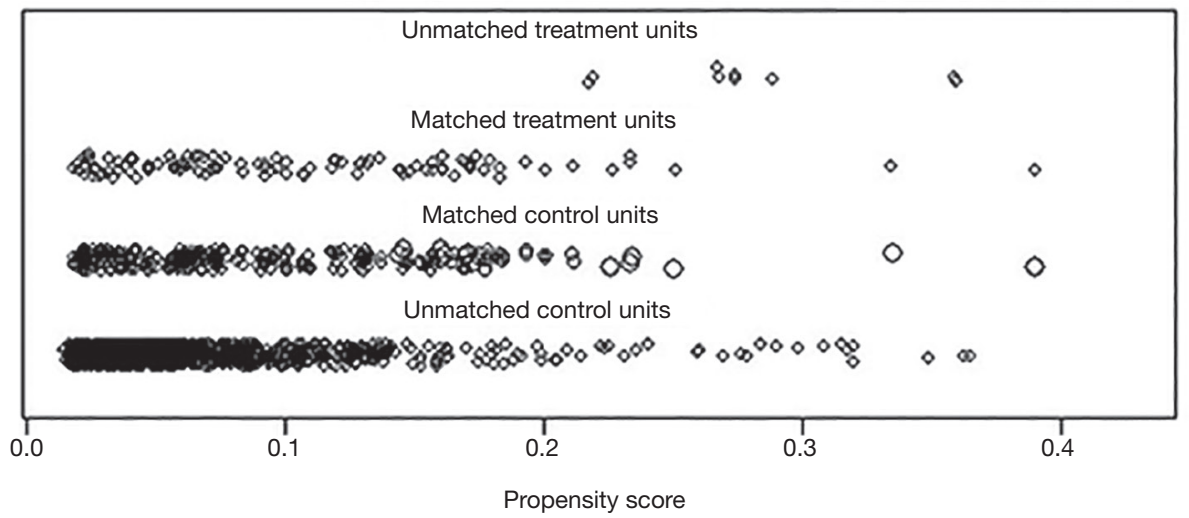

Figure S1 The characteristics of data before and after propensity score matching (Jitter).

Raw treated

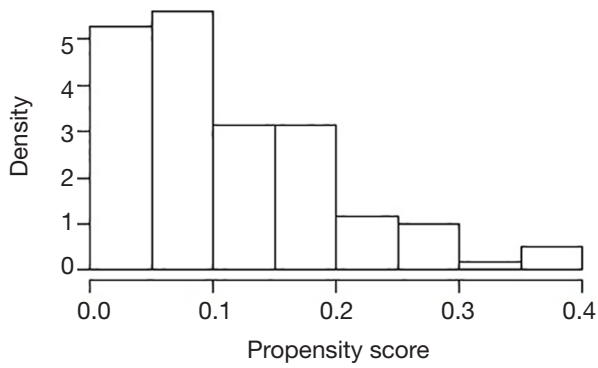

Raw control

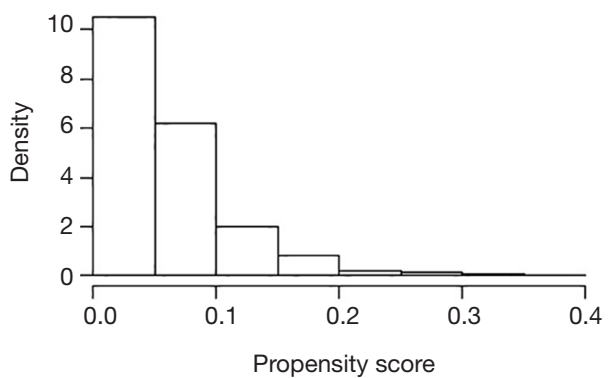

Matched treated

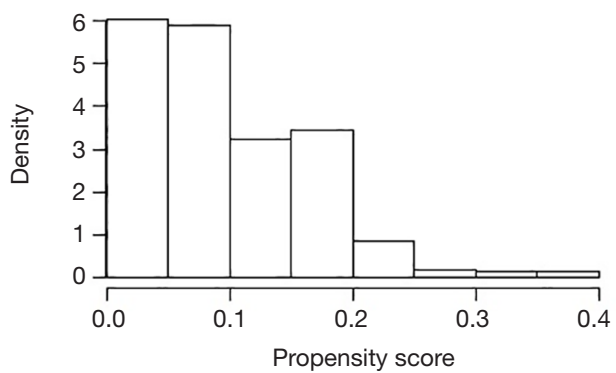

Matched control

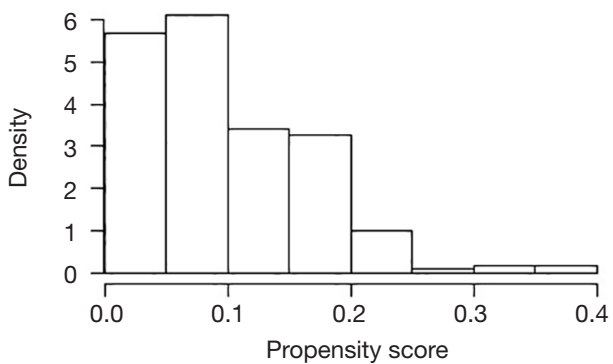

Figure S2 The characteristics of data before and after propensity score matching (Hist). 

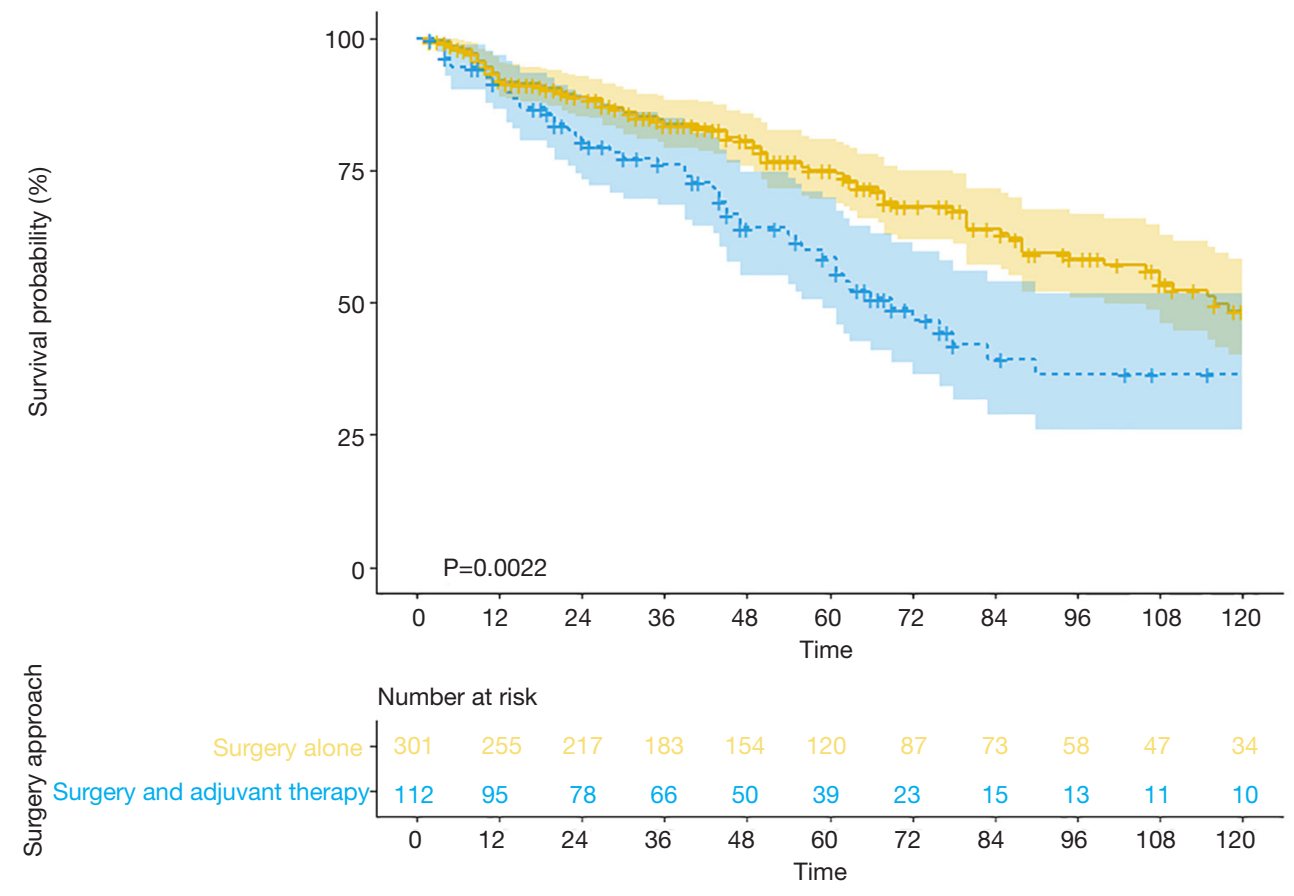

Surgery approach $\div$ Surgery $\div$ Surgery and adjuvant therapy

Figure S3 The overall survival of patients received surgery with or without adjuvant chemotherapy after propensity score matching.

Table S3 Mucinous adenocarcinoma of the lung patients overall survival after propensity score matching

\begin{tabular}{lccc}
\hline \multirow{2}{*}{ Group } & & Overall survival & \\
\cline { 2 - 4 } & Median (months) & $95 \% \mathrm{Cl}$ & $\mathrm{P}$ value \\
\hline Surgery and adjuvant therapy $(\mathrm{n}=112)$ & 69 & $59-144$ & 0.002 \\
Surgery alone $(\mathrm{n}=301)$ & 116 & $100-166$ & \\
\hline
\end{tabular}

